\title{
Respiratory infections associated with nontuberculous mycobacteria in non-HIV patients
}

\author{
M. Dailloux*, M.L. Abalain*, C. Laurain*, L. Lebrun`, C. Loos-Ayav*, A. Lozniewski*, \\ J. Maugein ${ }^{+}$and the French Mycobacteria Study Group
}

ABSTRACT: The incidence of nontuberculous mycobacteria (NTM) pulmonary diseases in HIVnegative patients was studied prospectively from January 1, 2001 to December 31, 2003 by 32 sentinel sites distributed throughout France.

In total, 262 patients who yielded NTM isolates from respiratory clinical specimens, met the bacteriological, radiological and clinical criteria established by the American Thoracic Society for NTM respiratory disease. Among the 262 NTM isolates, 234 were slow-growing mycobacteria (125 Mycobacterium avium-intracellulare complex (MAC), 66 M. xenopi, 34 M. kansasii) and 28 were rapidly growing mycobacteria (25 $M$. abscessus complex). In the Paris area, M. xenopi was the most frequently isolated species, followed by MAC.

Most patients (>50\%), except those with $M$. kansasii, had underlying predisposing factors such as pre-existing pulmonary disease or immune deficiency. Asthenia, weight loss, chronic cough and dyspnoea were the most common clinical symptoms. The classical radiological appearance of NTM infections was indistinguishable from that observed in patients with pulmonary tuberculosis.

In summary, the incidence of nontuberculous mycobacteria pulmonary infections in HIVnegative patients was estimated at $0.74,0.73$ and 0.72 cases per 100,000 inhabitants in 2001, 2002 and 2003, respectively.

KEYWORDS: HIV-negative patients, nontuberculous mycobacteria, pulmonary diseases

A s nontuberculous mycobacteria (NTM) are common in the environment, infection and colonisation by NTM are not easily distinguishable [1]. For this reason, the minimal criteria required for the diagnosis of pulmonary infection have been established [2,3]. NTM cause clinically significant infections in both immunosuppressed and immunocompetent patients [4, 5]. However, it has recently been shown that NTM infections are increasing in patients with chronic respiratory diseases [6]. Moreover, increased rates of NTM infections have been reported in areas in which the bacille CalmetteGuerin vaccination has been stopped due to the prevalence of tuberculosis [6, 7]. In contrast to tuberculosis, which is a disease of obligatory declaration in France and for which the annual incidence is therefore well known, the incidence of NTM infections in France is unknown due to a lack of systematic reporting. To determine the occurrence of NTM infections in France and

$\overline{\text { For editorial comments see page } 1076 .}$ to compare it with that of tuberculosis, data collected by a voluntary-based laboratory network, the French Mycobacteria Study Group (FMSG), was used. The present authors have previously published a study on Mycobacterium avium and $M$. intracellulare [8], and the aim of the current study was to extend this work to include all pulmonary infections caused by opportunistic mycobacteria. For this purpose, demographic, epidemiological, clinical and radiological data obtained over a 3-yr period, in 262 HIV-negative patients with NTM pulmonary infections, were prospectively analysed. Further, the incidence of NTM disease was determined taking into consideration the incidence of tuberculosis in France.

\section{MATERIALS AND METHODS}

The FMSG constitutes 32 sentinel sites distributed all over France; 21 in regional cities and 11 in the Paris area. This group was formed to provide a systematic surveillance of $M$. tuberculosis drug resistance and covers $\sim 25 \%$ of tuberculosis cases in France [8, 9]. Between January 1, 2001 and
AFFILIATIONS

${ }^{*} \mathrm{CHU}$, Nancy,

${ }^{\#} \mathrm{CHU}$, Brest,

"CHU, Clamart, Paris, and

${ }^{+}$CHU Haut-Lévêque, Bordeaux, France.

CORRESPONDENCE

M. Dailloux

Laboratoire de Bactériologie

Hôpital Brabois

Rue du Morvan

54500 Vandoeuvre les Nancy

France

Fax: 33383154376

E-mail: m.dailloux@chu-nancy.fr

Received:

May 112006

Accepted after revision:

October 132006 
December 31, 2003, the number of tuberculosis and NTM infections was recorded annually by the FMSG network covering the same population, therefore limiting the risk of differential reporting. For mycobacterial investigations, smears of respiratory secretions were stained and examined for the presence of acid-fast bacilli. Depending on the laboratories, samples of specimens were inoculated onto Löwenstein-Jensen medium (BioRad, Marnes La coquette, or bioMérieux, Marcy l'Etoile, France) and/or in Middlebrook broth medium (Becton Dickinson, NJ, USA or bioMérieux), and incubated, according to underlying conditions, at $30^{\circ} \mathrm{C}$ and $37^{\circ} \mathrm{C}$ or $40^{\circ} \mathrm{C}$ for $>2$ months. The identification of isolates was performed using approved methods, including biochemical tests and/or molecular tests such as DNA probes or genomic typing [10].

The laboratory records of the 32 sentinel sites were prospectively reviewed to identify patients in whom NTM were isolated from pulmonary specimens obtained during the study period. The bacteriological criteria defined by the American Thoracic Society (ATS) were used to select the patient records.

The data collected were: age, sex and underlying disorders such as pre-existing pulmonary disease, cystic fibrosis (CF), previous infection with $M$. tuberculosis or NTM, leukaemia and/or carcinoma, and immunosuppressive therapy and/or transplantation. Pulmonary (presence of cough, dyspnoea, haemoptysis) and systemic (presence of fever, night sweats, weight loss and asthenia) clinical manifestations, as well as radiographic data (presence of nodules, infiltrates, cavitations) were collected at the time of diagnosis. Patients with active tuberculosis and/or HIV-positive patients were excluded from this study.

Pulmonary disease was established in patients when ATS-defined bacteriological criteria were associated with radiographic and clinical criteria suggesting mycobacterial infections and/or when a treatment was decided by a clinician.

Moreover, each laboratory provided the number of culturepositive tuberculosis cases observed during the study period. The data were compared using the Chi squared or t-test. A p-value of $<0.05$ was considered significant.

\section{RESULTS}

Over the study period, 262 HIV-negative patients with lung disease due to NTM were identified (table 1). Among these lung diseases, 234 (89\%) were due to slow-growing mycobacteria (SGM): the most common species was $M$. aviumintracellulare complex (MAC; 125 isolates) followed by $M$. xenopi (66 isolates) and M. kansasii (34 isolates), while M. malmoense, M. simiae, M. szulgai, M. scrofulaceum and $M$. triplex were rarely isolated (nine isolates). Among the 28 infections $(10.6 \%)$ caused by rapidly growing mycobacteria (RGM), 25 were due to $M$. abscessus complex, 12 of which were isolated in patients with CF. The other RGM species ( $M$. fortuitum, M. smegmatis, $M$. peregrinum) were only isolated once. The low number of NTM cases reported by each sentinel site does not allow analysis of distribution according to the regional cities; however, the Paris area was examined individually from all the other French regions. As reported in table 2, the rate of patients with $M$. xenopi diseases was significantly higher $(\mathrm{p}<0.001)$ in the Paris area $(40.5 \%)$ than in regional cities $(18 \%)$, while the proportion of MAC was lower in the Paris area $(\mathrm{p}<0.01)$. For M. kansasii and M. abscessus infections, similar rates were observed in the Paris area and in all regional cities combined (table 2).

Assuming that the incidence of tuberculosis cases in France for 2001 is 10.8 per 100,000 inhabitants $[11,12,13]$, the annual population studied by the FMSG was evaluated to be 11.3 million $\left(1,225 / 10.8 \times 100,000=11.3 \times 10^{6}\right)$. Thus, overall national rates of NTM pulmonary disease were estimated at $0.74,0.73$ and 0.72 cases per 100,000 inhabitants in 2001, 2002 and 2003, respectively (table 3).

The main characteristics of patients with mycobacterial infections are listed in table 4. Male patients were more frequently infected by $M$. xenopi or M. kansasii (77.3 and $79.4 \%$, respectively; $p=0.001)$ whereas female patients were more frequently infected with MAC or RGM (62.4 and $68 \%$, respectively; $p=0.001)$. The mean age was $>60 \mathrm{yrs}$, except for patients infected by $M$. kansasii (54 yrs). Predisposing factors for developing lung disease were frequently found among patients with NTM infections, with pre-existing pulmonary diseases being the most frequent. However, such factors were significantly less frequent in patients infected with $M$. kansasii than in those infected with other species $(\mathrm{p}<0.0001)$.

Interstital infiltrates and/or nodules were the predominant radiological patterns, with no significant difference between patients infected by different species. A higher rate of cavitations (38\%) was observed in patients infected by $M$. kansasii. Constitutional symptoms, including asthenia and weight loss, were common; most patients presented cough and dyspnoea. Haemoptysis occurred in $<26 \%$ of patients.

\section{DISCUSSION}

In the present study, the mean number of respiratory infections due to NTM in HIV-negative patients was $87 \cdot \mathrm{yr}^{-1}$ and was stable over the 3-yr study period. MAC was preponderantly isolated, followed by M. xenopi, M. kansasii and M. abscessus. During the study period, no significant changes in the incidence rates of NTM pulmonary infections in HIV-negative patients $(0.74,0.73$ and 0.72 cases per 100,000 inhabitants in 2001, 2002 and 2003, respectively) were observed. These rates are similar to those observed in Europe but lower than those reported in the USA [6].

\begin{tabular}{|c|c|c|c|c|c|c|c|}
\hline \multirow[t]{3}{*}{ TABL } & LE 1 & \multicolumn{6}{|c|}{$\begin{array}{l}\text { Number of nontuberculous mycobacteria } \\
\text { infections by species in HIV-negative patients }\end{array}$} \\
\hline & \multicolumn{4}{|c|}{ Slow growing } & \multicolumn{2}{|c|}{$\begin{array}{l}\text { Rapidly } \\
\text { growing }\end{array}$} & \multirow[t]{2}{*}{ Total } \\
\hline & MAC & M. xenopi & M. kansasii & Others & CF & Non-CF & \\
\hline 2001 & 42 & 25 & 6 & 2 & 5 & 4 & 84 \\
\hline 2002 & 44 & 17 & 16 & 4 & 4 & 6 & 91 \\
\hline 2003 & 39 & 24 & 12 & 3 & 7 & 2 & 87 \\
\hline Total & $125(47.7)$ & 66 (25.2) & 34 (12.9) & $9(3.4)$ & $16(6.1)$ & $12(4.6)$ & 262 \\
\hline \multicolumn{8}{|c|}{$\begin{array}{l}\text { Data are presented as } \mathrm{n} \text { or } \mathrm{n}(\%) \text {, unless otherwise stated. MAC; } \\
\text { Mycobacterium avium-intracellulare complex; } M \text {. xenopi: Mycobacterium xenopi; } \\
\text { M. kansasii: Mycobacterium kansasii; CF: cystic fibrosis. }\end{array}$} \\
\hline
\end{tabular}




\begin{tabular}{lcccc}
\hline TABLE 2 & $\begin{array}{l}\text { Number of patients with nontuberculous } \\
\text { mycobacteria (NTM) infections (total number and } \\
\text { distribution according to species) or with } \\
\text { tuberculosis according to geographical area }\end{array}$ \\
& Total $\mathbf{n}$ & Paris area & Regional cities & p-value \\
\hline NTM infections n & 263 & 84 & 179 & \\
MAC & 125 & 32.1 & 54.7 & $<0.01$ \\
M. xenopi & 66 & 40.5 & 18.4 & $<0.001$ \\
M. kansasii & 34 & 16.7 & 11.2 & $>0.05$ \\
M. abscessus & 24 & 7.1 & 10.1 & $>0.05$ \\
Other NTM & 13 & 3.6 & 5.6 & $>0.05$ \\
Tuberculosis n & 3787 & 1250 & 2537 & \\
\hline
\end{tabular}

Data are presented as \%, unless otherwise stated. MAC: $M$. avium-intracellulare complex; M. xenopi: Mycobacterium xenopi; M. kansasii: Mycobacterium kansasii; M. abscessus: Mycobacterium abscessus.

Among patients infected by SGM, MAC pulmonary disease was found to be more frequent in elderly females (62\%). This is in accordance with the observations of HAN et al. [14], who recently reported a higher isolation rate of MAC in females, in whom an age trend for the isolation of $M$. intracellulare was observed. The predominance of females among these patients may be related to specific immune deficiencies and/or the habit of voluntary suppression of cough, particularly in older females, such as those suffering from Lady Windermere syndrome [14, 15]. FIELD et al. [16] noted that until 1980 patients with MAC pulmonary infections were predominantly male, and this disease has now become more prevalent in elderly females over the last decade. In the present study, in line with findings of previous studies [17, 18], M. kansasii and M. xenopi were mostly isolated in males and the mean age of patients infected by $M$. kansasii (54 yrs) was lower than that of patients infected by M. xenopi (60 yrs). Excluding CF patients, the mean age of patients infected by RGM was 62 yrs and the sex ratio was 2.5 female/male. This is in concordance with previous reports that showed a female predominance for RGM diseases [19]. It has been shown that the prevalence of NTM infections is higher in CF adults than in CF children [20, 21]. In the current study, no NTM infection was observed in CF patients aged $>30$ yrs.

NTM infections usually occurred in patients with predisposing host factors such as impairment of local pulmonary defences or generalised immune defects $[6,8,22,23]$. However, HENRY et al. [4] reported that among patients who developed significant NTM pulmonary infections, $37 \%$ had no underlying lung disease. In the present study, $44 \%$ of $M$. kansasii infections occurred in patients without risk factors. Similar results were reported by BLOCH et al. [24]. This may be explained by the fact that some subtypes of $M$. kansasii, such as subtype 1, possess virulence factors that enable them to cause disease in immunocompetent individuals [25, 17]. In the current study, this remains to be characterised as no genotyping was performed. A higher rate of predisposing factors $(\sim 85 \%)$ was observed among patients infected by species other than $M$. kansasii. Among these patients with predisposing conditions, $\sim 50 \%$ had pre-existing chronic lung diseases, such as chronic

\begin{tabular}{lcccc} 
TABLE 3 & $\begin{array}{l}\text { Number of tuberculosis and nontuberculous } \\
\text { mycobacteria (NTM) lung infections observed by } \\
\text { the French Mycobacteria Group }\end{array}$ \\
& Tuberculosis & $\begin{array}{c}\text { NTM } \\
\text { infections }\end{array}$ & $\begin{array}{c}\text { Tuberculosis } \\
\text { incidence }\end{array}$ & $\begin{array}{c}\text { NTM } \\
\text { incidence }\end{array}$ \\
\hline $\mathbf{2 0 0 1}$ & 1225 & 84 & 10.8 & 0.74 \\
$\mathbf{2 0 0 2}$ & 1319 & 91 & 10.5 & 0.73 \\
$\mathbf{2 0 0 3}$ & 1244 & 87 & 10.2 & 0.72 \\
\hline \#: per 100,000 inhabitants. & & & \\
\end{tabular}

obstructive pulmonary disease (COPD), and 25\% had been previously diagnosed with tuberculosis. Immunosuppressive therapies, haematopoietic malignancies or extra-pulmonary neoplasms, which are also major risk factors for the development of NTM disease, were found in $\sim 20 \%$ of the current patients. These findings are similar to those previously published by AKSAMIT [22] who related the importance of pre-existing lung disease (COPD, bronchectasis, prior tuberculosis etc.) to the development of a MAC infection in nonimmunocompromised patients. Similar observations were made for M. xenopi-infected patients by JENKINS et al. [18], who reported that a pre-existing lung disease or an impaired immune response was observed in two-thirds and one-quarter of these patients, respectively. Several studies found that $\mathrm{CF}$ may have an impact on the development of NTM infections [20]. In the present study, of the 14 CF patients, 12 had an infection caused by M. abscessus and two had an infection due to MAC.

Available clinical investigations indicate that systemic and pulmonary symptoms are associated with NTM infections, although it is often difficult to distinguish them from those related to the underlying lung diseases [5]. In the current study, the most frequent systemic symptoms were asthenia or weight loss. The most frequent pulmonary symptoms were chronic cough, followed by dyspnoea and haemoptysis. Haemoptysis was present in $25 \%$ of SGM-infected patients and was rarely observed in patients with RGM infections.

Various radiographic features have been associated with NTM lung infections. However, interpretation of chest radiographs is often difficult, as the radiographic appearance of NTM disease is indistinguishable from that seen with pulmonary tuberculosis and because chronic underlying pulmonary disease may interfere. In the present study, the most common abnormality was the presence of infiltrates that were observed in one-third and one-half of patients infected respectively by SGM and RGM. Cavities were also found to be more frequently observed in M. kansasii infections (38.2\%) than in other infections. JENKINS et al. [18] reported cavities as being the most frequent radiological pattern in patients infected by $M$. xenopi. This was not observed in the present study. This discrepancy may be the result of differences in the populations studied and/or in the study designs. It was reported that $M$. abscessus lung diseases radiographically resemble MAC lung diseases and that for this species the nodular infiltrates were the predominant abnormalities [19, 22]. 


\begin{tabular}{|c|c|c|c|c|c|}
\hline & MAC & M. xenopi & M. kansasii & RGM/non-CF & p-value \\
\hline Age yrs & $70(1-95)$ & $60(19-84)$ & $54(22-93)$ & $62(32-81)$ & $<0.0001$ \\
\hline Sex male/female & $53 / 80$ & $52 / 15$ & $27 / 7$ & $5 / 11$ & 0.001 \\
\hline \multicolumn{6}{|l|}{ Underlying conditions } \\
\hline Previous M. tuberculosis & $35(28)$ & $17(25.8)$ & $9(26.5)$ & $3(18.7)$ & 0.88 \\
\hline Immunosupression/transplantation & $34(27.7)$ & $17(25.8)$ & $5(14.7)$ & $1(6.2)$ & 0.15 \\
\hline None/anorexia & $14(11.2)$ & $18(27.3)$ & $15(44.1)$ & $4(25)$ & 0.0002 \\
\hline \multicolumn{6}{|l|}{ Radiographic abnormalities } \\
\hline Infiltrates & $46(36.8)$ & $21(31.8)$ & $12(35.3)$ & $8(50)$ & 0.59 \\
\hline Nodules & $28(22.4)$ & $21(31.8)$ & $10(29.4)$ & $3(18.7)$ & 0.44 \\
\hline Weight loss & $60(49)$ & $28(42.4)$ & $15(44.1)$ & $13(81.3)$ & 0.043 \\
\hline Asthenia & $73(58.4)$ & $31(47)$ & $17(50)$ & $13(81.8)$ & 0.069 \\
\hline No symptoms & $35(28)$ & $22(33.3)$ & $10(29.4)$ & $0(0)$ & 0.065 \\
\hline \multicolumn{6}{|l|}{ Pulmonary symptoms } \\
\hline Cough & $97(77.6)$ & $39(59.1)$ & $22(64.7)$ & $12(75)$ & 0.048 \\
\hline Haemoptysis & $18(14.4)$ & $17(25.8)$ & $8(23.5)$ & $2(12.5)$ & 0.20 \\
\hline Dyspnoea & $60(48)$ & $37(56.7)$ & $9(26.5)$ & $6(37.5)$ & 0.036 \\
\hline No symptoms & $12(9.6)$ & $12(18.2)$ & 7 (20.5) & $0(0)$ & 0.071 \\
\hline
\end{tabular}

Data are presented as $\mathrm{n}$, mean (range) or $\mathrm{n}(\%)$, unless otherwise stated. MAC: Mycobacterium avium-intracellulare complex; $M$. xenopi: Mycobacterium xenopi; $M$. kansasii: Mycobacterium kansasii; RGM: rapidly growing mycobacteria; $\mathrm{CF}$ : cystic fibrosis.

Recommendations have been established for the treatment of lung infection caused by NTM [2, 3]. Treatment with rifampicin plus ethambutol, with or without isoniazid, is currently recommended for lung disease caused by M. kansasii $[3,24,26,27]$. During the present study, patients infected with M. kansasii were predominantly treated with rifampicin, ethambutol and isoniazid for 9 months, and only one relapse occurred. Patients with pulmonary MAC or M. xenopi disease are usually treated with multidrug regimens including rifampicin or rifabutin, ethambutol, isoniazid and clarithromycin $[4,28,29]$. In the current study, high mycobacterial clearance rates were observed when patients were treated with this combination plus another antibiotic, such as a fluoroquinolone. However, relapse rates were high for several months or years, even after conversion of sputum culture from positive to negative. Among patients with M. xenopi infections, $22.8 \%$ had been diagnosed with the same infection a few years earlier. JENKINS et al. [18] reported a relapse rate of $12 \%$ for patients infected by $M$. xenopi, treated in a randomised trial with rifampicin plus ethambutol, rifampicin, or ethambutol plus isoniazid. Thus, for patients with localised pulmonary lesions persisting after medical treatment, a surgical treatment is recommended [30]. Antimicrobial treatment of lung disease caused by $M$. abscessus is usually unsuccessful; therefore, the ATS recommends the use of antimicrobial drugs, such as clarithromycin plus cefoxitin and/or amikacin. However, acquired resistance to clarithromycin has been reported in $M$. abscessus and may cause eradication failure [19, 31].
In summary, the clinical presentations and radiographic manifestations of pulmonary nontuberculous mycobacteria infections are quite variable and are further modified by underlying comorbidities. To establish the diagnosis of infection it is necessary to combine data obtained from the mycobacterial laboratory with the clinician's assessments. Further, the adherence to published guidelines should improve the diagnosis of these diseases and consequently their survey all over the world. The present study indicates that the French Mycobacteria Study Group could provide an estimation of the frequency of nontuberculous mycobacteria disease in France, particularly now, as the bacille CalmetteGuerin vaccine is less frequently used due to the low risk of tuberculosis.

\section{ACKNOWLEDGEMENTS}

The members of the French Mycobacteria Study Group are as follows: R. Bauriaud (Toulouse), P. Bemer (Nantes), A. Bourgoin (Poitiers), L. Brasme (Reims), E. Cambau (Créteil), A. Carricajo (Saint-Etienne), C. Carriere (Montpellier), B. Cattier (Tours), S. Coignard (Paris), M. Chomarat (Lyon), G. Couetdic (Besançon), M.F. David (Paris), L. Deforges (Créteil), F. DoucetPopulaire (Versailles), J.M. Duez (Dijon), M.C. Gutierrez (Paris), J.L. Hermann (Paris), V. Jarlier (Paris), V. Lalande (Paris), L. Landraud (Nice), N. Lemaitre (Lille), B. Malbruny (Caen), C. Martin (Limoges), C. Offredo (Paris), M. Pestel-Caron (Rouen), J.P. Romaszko (Clermont-Ferrand), E. Ronco (Garches), 
A. Rossier (Paris), D. Sicard (Nice), D. Terru (Montpellier), C. Truffot-Pernot (Paris), A. Vachée (Roubaix), V. Vincent (Paris).

\section{REFERENCES}

1 Catanzaro A. Diagnosis, differentiating colonization, infection and disease. Clin Chest Med 2002; 23: 599-601.

2 American Thoracic Society. Diagnosis and treatment of disease caused by nontuberculous mycobacteria. Am J Respir Crit Care Med 1997; 156: S1-S25.

3 Subcommitee of the Joint Tuberculosis Committee of the British Thoracic Society. Management of opportunist mycobacterial infections. Joint tuberculosis committee guidelines 1999. Thorax 2000; 55: 210-218.

4 Henry MT, Inamdar L, O'Riordain D, Schweiger M, Watson JP. Nontuberculous mycobacteria in non-HIV patients: epidemiology, treatment and response. Eur Respir J 2004; 23: 741-746.

5 Wagner D, Young LS. Nontuberculous mycobacterial infections: a clinical review. Infection 2003; 31: 257-270.

6 Marras TK, Daley CL. Epidemiology of human pulmonary infection with nontuberculous mycobacteria. Clin Chest Med 2002; 23: 553-567.

7 Valadas E. Nontuberculous mycobacteria: clinical importance and relevance to Bacille Calmette-Guérin vaccination. Clin Infect Dis 2004; 39: 457-458.

8 Maugein J, Dailloux M, Carbonnelle B, et al. Sentinel-site surveillance of Mycobacterium avium complex pulmonary disease in France. Eur Respir J 2005; 26: 1-4.

9 Robert J, Trystram D, Truffot-Pernot C, Carbonnelle B, Grosset J. Surveillance of Mycobacterium tuberculosis drug resistance in France 1995-1997 Azay mycobacteria study group. Int J Tuberc Lung Dis 2000; 4: 665-672.

10 Martinez V, Gicquel B. Laboratory diagnosis of mycobacterial infections. Arch pédiatrie 2005; 12: S96-S101.

11 Cailhol J, Che D, Campese C, Decludt B. Les cas de tuberculose déclarés en France en 2001. [Cases of tuberculosis reported in France in 2001.] Bull Epidemiol Hebdo 2003; 10-11: 54-57.

12 Che D, Campese C, Decludt B. Les cas de tuberculose déclarés en France en 2002. [Cases of tuberculosis reported in France in 2002.] Bull Epidemiol Hebdo 2004; 4: 13-16.

13 Che D, Bitar D. Les cas de tuberculose déclarés en France en 2003. [Cases of tuberculosis reported in France in 2003.] Bull Epidemiol Hebdo 2005; 17-18: 66-69.

14 Han XY, Tarrand JJ, Infante R, Jacobson KL, Truong M. Clinical significance and epidemiologic analyses of Mycobacterium avium and Mycobacterium intracellulare among patients without AIDS. J Clin Microbiol 2005; 43: 4407-4412.

15 Dhillon SS, Watanakunakorn C. Lady Windermere Syndrome: Middle lobe bronchiectasis and Mycobacterium avium complex infection due to voluntary cough suppression. Clin Infec Dis 2000; 30: 572-575.

16 Field SK, Fisher D, Cowie RL. Mycobacterium avium complex pulmonary disease in patients without HIV infection. Chest 2004; 126: 566-581.
17 Arend SM, Cerda de Palou E, de Haas P, et al. Pneumonia caused by Mycobacterium kansasii in a series of patients without recognised immune defect. Clin Microbiol Infect 2004; 10: 738-748.

18 Jenkins PA, Campbell IA. Research committee of the British Thoracic Society. Pulmonary disease caused by Mycobacterium xenopi in HIV-negative patients: five year follow-up of patients receiving standardised treatment. Respir Med 2003; 97: 439-444.

19 Daley CL, Griffith DE. Pulmonary disease caused by rapidly growing mycobacteria. Clin Chest Med 2002; 23: 623-632.

20 Olivier KN, Weber DJ, Wallace RJ Jr, et al. Nontuberculous mycobacteria I: multicenter prevalence study in cystic fibrosis. Am J Respir Crit Care Med 2003; 167: 828-834.

21 Pierre-Audigier C, Ferroni A, Sermet-Gaudelus I, et al. Age-related prevalence and distribution of nontuberculous mycobacterial species among patients with cystic fibrosis. J Clin Microbiol 2005; 43: 3467-3470.

22 Aksamit TR. Mycobacterium avium complex pulmonary disease in patients with pre-existing lung disease. Clin Chest Med 2002; 23: 643-653.

23 McGarvey J, Bermudez LE. Pathogenesis of nontuberculous mycobacteria infection. Clin Chest Med 2002; 23: 569583.

24 Bloch KC, Zwerling L, Pletcher MJ, et al. Incidence and clinical implications of isolation of Mycobacterium kansasii: results of a 5-year, population-based study. Ann Intern Med 1998; 129: 698-704.

25 Taillard C, Greub G, Weber R, et al. Clinical implications of Mycobacterium kansasii species heterogeneity: Swiss National Survey. J Clin Microbiol 2003; 41: 1240-1244.

26 Research Committee of the British Thoracic Society. Mycobacterium kansasii pulmonary infections: a prospective study of the results of nine months of treatment with rifampicin and ethambutol. Thorax 1994; 49: 442-445.

27 Griffith DE. Management of disease due to Mycobacterium kansasii. Clin Chest Med 2002; 23: 613-621.

28 May T, Brel F, Beuscart C, et al. Comparison of combination therapy regimens for treatment of human immunodeficiency virus-infected patients with disseminated bacteremia due to Mycobcterium avium. Clin Infect Dis 1997; 25: 621-629.

29 Research Committee of the British Thoracic Society. First randomised trial of treatments for pulmonary disease caused by $M$ avium intracellulare, $M$ malmoense, and $M$ xenopi in HIV negative patients: rifampicin, ethambutol and isoniazid versus rifampicin and ethambutol. Thorax 2001; 56: 167-172.

30 Lang-Lazdunski L, Offredo C, Le Pimpec-Barthes F, Danel C, Dujon A, Riquet M. Pulmonary resection for Mycobacterium xenopi pulmonary infection. Ann Thorac Surg 2001; 72: 1877-1882.

31 Brown-Elliot BA, Wallace RJ. Clinical and taxonomic status of pathogenic nonpigmented or late-pigmented rapidly growing mycobacteria. Clin Microbiol Rev 2002; 15: 716-746. 